\title{
Erosion and deposition on JET divertor and limiter tiles during the experimental campaigns 2005-2009
}

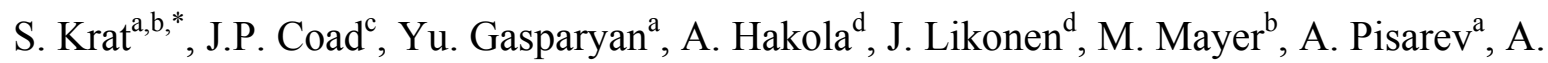 \\ Widdowson $^{\mathrm{c}}$, and JET-EFDA contributors \\ JET-EFDA, Culham Science Centre, Abingdon, OX14 3DB, UK
}

${ }^{a}$ National Research Nuclear University “MEPhI”, Moscow Kashirskoe road 31, 115409, Russia;.

${ }^{b}$ Max-Planck-Institut für Plasmaphysik, EURATOM Association, Boltzmannstr. 2, 85748 Garching, Germany.

${ }^{c}$ Culham Science Centre, EURATOM/UKAEA - Fusion Association, Abingdon, Oxfordshire OX14 3DB, UK.;

${ }^{d}$ Association EURATOM-Tekes, Technical Research Centre of Finland, PO Box 1000, FI-02044 VTT, Finland.

\begin{abstract}
Erosion from and deposition on JET divertor tiles used during the 2007-2009 campaign and on inner wall guard limiter (IWGL) tiles used during 2005-2009 are studied. The tungsten coating on the divertor tiles was mostly intact with the largest erosion $\sim 30 \%$ in a small local area. Locally high erosion areas were observed on the load bearing divertor tile 5 and on the horizontal surface of the divertor tile 8 . The IWGL tiles show a complicated distribution of erosion and deposition areas. The total amount of carbon deposited on the all IWGL tiles
\end{abstract}


during the campaign $2005-2009$ is estimated to be $65 \mathrm{~g}$. The density of carbon deposits is estimated to be $0.67-0.83 \mathrm{~g} / \mathrm{cm}^{3}$.

PACS: 52.40Hf, 52.55.Fa, 82.80.Yc

PSI-20 keywords: JET, Erosion \& Deposition, Divertor, Limiter

*corresponding author mail address: the Russian Federation, Moscow, Kahirskoe shosse, 31, 115409

*corresponding author e-mail: stepan.krat@gmail.com

Presenting author: Stepan Krat

Presenting author e-mail: stepan.krat@gmail.com 


\section{Introduction}

Erosion of plasma facing materials (PFM) and subsequent re-deposition are critical processes for the operation of fusion devices. Erosion determines the lifetime of plasma facing components (PFCs), re-deposition of eroded material on PFCs and in shadowed areas may lead to an increase of the tritium inventory via co-deposition. It was already observed at JET, that outer divertor tiles are net erosion dominated areas [1,2], while thick deposits are found in the inner divertor [2,3,4]. Main chamber limiter tiles analysed after the Mark I Be divertor campaign showed a complicated distribution of erosion/deposition areas [5], but net erosion/deposition could not be analyzed quantitatively on these tiles. Net carbon erosion on the outer and net carbon deposition on the inner divertor was also observed during the carbondominated operational phases of ASDEX Upgrade [6,7].

During the investigated experimental campaigns JET was operating as a full carbon machine with regular beryllium evaporations. In this work the erosion of a tungsten marker and deposition of carbon and beryllium were quantitatively investigated on the selected outer divertor tiles exposed during the 2007-2009 campaigns and on inner wall guard limiter (IWGL) tiles exposed during 2005-2009 by means of Rutherford Backscattering Spectroscopy (RBS).

2. Experimental details

\subsection{Divertor tiles}

One load bearing tile (LBT), and tiles G7B and G8A from the vertical outer divertor have been analyzed. Their positions are shown on fig. 1. The tiles were installed in the divertor from 2007 to 2009. The tiles were made of Carbon Fiber Composite (CFC) and were coated with a W/Re multilayer using physical vapor deposition (PVD). The nominal thicknesses of 
the coating were $6 \mu \mathrm{m} \mathrm{Re}+2 \mu \mathrm{m} \mathrm{W}+2 \mu \mathrm{m} \mathrm{Re}+4 \mu \mathrm{m} \mathrm{W}$, in total $14 \mu \mathrm{m}$. W and Re cannot be distinguished by RBS and have almost identical stopping powers and kinematics, in this paper they are approximated to be one single thick W layer. These coatings were not precharacterized by RBS, but their thicknesses were measured by scanning electron microscopy of cross-sections deposited on witness samples.

\subsection{Limiter tiles}

Two IWGL tiles, 3X11L and 3X11R, have been analyzed, these are tiles located at the mid-plane of the inner wall limiter. The tiles were exposed in JET from 2005 to 2009. The tiles were made of CFC and were coated with two marker stripes; one with tungsten and the other with carbon. The thickness of the marker stripes was measured using RBS before their installation in JET. The thickness of the $\mathrm{W}$ marker was $2 \times 10^{19}$ atoms $/ \mathrm{cm}^{2}$ (about $3 \mu \mathrm{m}$ ). The carbon marker consisted of a thin W layer with a thickness of $6 \times 10^{18}$ atoms $/ \mathrm{cm}^{2}$ and a $\mathrm{C}$ layer with a thickness of $1 \times 10^{20}$ atoms $/ \mathrm{cm}^{2}$ (about $9 \mu \mathrm{m}$ ) on the top. The $\mathrm{W}$ layer marked the initial CFC surface.

\subsection{RBS analysis}

The tiles were analyzed using RBS in the BOMBARDINO facility at IPP Garching. The analysis was performed by using $4.5 \mathrm{MeV} \mathrm{H}^{+}$ions with the detector positioned at $165^{\circ}$ in the laboratory measurement system. This energy was chosen to ensure that the sampling depth can exceed the thickness of the W-Re layer on the divertor tiles. The same ion energy was used for the IWGL tiles to avoid additional calibrations of the proton detector. Each measurement was conducted with $5 \mu \mathrm{C}$ of accumulated charge of ion current. The spectra obtained were analyzed using the SIMNRA program [8]. SRIM 2010 stopping powers and

non-Rutherford backscattering cross-section data from [9-11] were used for ${ }^{12} \mathrm{C},{ }^{13} \mathrm{C},{ }^{16} \mathrm{O}$, 
respectively. The cross sections for ${ }^{12} \mathrm{C},{ }^{16} \mathrm{O}$ are for an interaction angle of $165^{\circ}$ and the cross section for ${ }^{13} \mathrm{C}$ is for an interaction angle of $163.8^{\circ}$.

\section{Results and Discussion}

\subsection{Divertor tiles}

The thickness of $\mathrm{W}$ coatings remained on the LBT divertor tile is shown in Fig. 2. On average the remaining thickness of the $\mathrm{W}$-Re multilayer was $1.1 \times 10^{20}$ atoms $/ \mathrm{cm}^{2}$. This is very close to the nominal initial thickness of the $\mathrm{W}$ coating, i.e. the tungsten erosion is low in most areas.

An intense area of erosion approximately $4 \mathrm{~cm}$ in width is observed on the central part of the LBT tile, from $\mathrm{s} \approx 1195 \mathrm{~mm}$ to $\mathrm{s} \approx 1235 \mathrm{~mm}$ (fig. 2). RBS spectra from this area of the tile shows a characteristic of intense inhomogeneous erosion [1], which is up to $30 \%$ of the initial coating thickness (fig. 2). The average thickness of the remaining tungsten coating is $8.8 \times 10^{19}$ atoms $/ \mathrm{cm}^{2}$ in this area. The RBS spectrum figure 3 shows a carbon edge and a tungsten edge which are both from the surface of the sample thus indicating that both elements are present at the surface. There are two possible explanations for this, (i) inhomogeneous erosion, i.e., tungsten is preferentially eroded in some areas revealing carbon underneath, (ii) tungsten carbide has been formed on the surface. Similar results have been observed in [1] as well as in [12] where Scanning Electron Microscopy (SEM) revealed the inhomogeneous erosion of a tungsten coating on a Load Bearing Tile exposed in JET from 2005 - 2007.

A small amount of deposition is observed on the outer part of the LBT tile, i.e., the edge furthest from the centre of the torus. The area of deposition starts at $\mathrm{s}=1240 \mathrm{~mm}$ and extends for $48 \mathrm{~mm}$ in the poloidal direction the end of the tile, $\mathrm{s}=1288 \mathrm{~mm}$. Both carbon and oxygen 
were detected on the surface of the tiles on top of the $\mathrm{W}$ coating with the amounts of up to $2.6 \times 10^{19} \mathrm{C}$-atoms $/ \mathrm{cm}^{2}$ and $2 \times 10^{18} \mathrm{O}$-atoms $/ \mathrm{cm}^{2}$.

At the end of the 2009 operations there was a tracer experiment where labeled methane ${ }^{13} \mathrm{CH}_{4}$ was injected just under the outer edge of the LBT providing ${ }^{13} \mathrm{C}$ tracer [13]. As a result of this experiment up to $1.5 \times 10^{18}$ atoms $/ \mathrm{cm}^{2}{ }^{13} \mathrm{C}$ was detected on the bottom half of tile 7 , similar to the result obtained in [14]. The area extended for $60 \mathrm{~mm}$, from s=1620 $\mathrm{mm}$ to $\mathrm{s} \approx 1680 \mathrm{~mm}$ from the bottom of the tile along the poloidal cross section (fig. 4). The area showed relatively homogeneous erosion of $\mathrm{W}$ with an average of $10^{20}$ atoms $/ \mathrm{cm}^{2} \mathrm{~W}$ remaining on the tile surface, i.e. around $90 \%$ of the original marker layer. Erosion in this region is slightly higher than in the rest of the tile (fig. 4). The distribution of ${ }^{13} \mathrm{C}$ on the surface of the tile does not fully correspond to the distribution of ${ }^{12}$ Clocated in the bottom part of the tile.

The middle part of tile G7B is the region of $\mathrm{W}$ erosion. The intensity of erosion extends for $40 \mathrm{~mm}$ and increases in the downward direction, i.e., from $\mathrm{s} \approx 1730 \mathrm{~mm}$ down to $\mathrm{s} \approx 1690$ $\mathrm{mm}$. The thickness of the remaining $\mathrm{W}$ layer decreases from $1.2 \times 10^{20}$ atoms $/ \mathrm{cm}^{2}$ to $10^{20}$ atoms $/ \mathrm{cm}^{2}$.

In contrast to tile 7, the horizontal surface of G8A tile ( $\mathrm{s}=2005 \mathrm{~mm}$ to $2053 \mathrm{~mm}$ in fig. 4) shows intense and inhomogeneous erosion, similar to the central area of LBT. The erosion intensity increases to the lower S-coordinates with the thickness of the remaining $\mathrm{W}$ layer decreasing from $1.1 \times 10^{20}$ atoms $/ \mathrm{cm}^{2}$ at the highest $(\mathrm{s}=2041 \mathrm{~mm})$ to $7.6 \times 10^{19}$ atoms $/ \mathrm{cm}^{2}$ at the (lowest $\mathrm{s}=2015 \mathrm{~mm}$ ). Other surfaces of the tile show low levels of erosion decreasing in the downward direction (fig. 4).

\subsection{IWGL tiles}


The IWGL tiles show a complicated distribution of erosion and deposition areas compared with that on divertor tiles, a schematic map of the distribution of these areas is shown in Fig. 5. The distribution is close to the ones observed in $[12,14,16]$. On both tiles the carbon marker stripe has totally disappeared in all areas close to the plasma. Probably this is not due to erosion, but due to the delamination of the stripe.

The outermost areas of the limiter tiles, which are farthest away from the plasma, are the least affected. They show only very small modifications by the plasma operation: little erosion and some very small amounts of deposits (Fig. 6). On the $3 \mathrm{X} 11 \mathrm{~L}$ (left) tile this area is $30 \mathrm{~mm}$ wide (-150 $\mathrm{mm}$ to $-120 \mathrm{~mm}$ in fig. 6) and on the $3 \mathrm{X} 11 \mathrm{R}$ (right) this region is $40 \mathrm{~mm}$ wide (110 $\mathrm{mm}$ to $150 \mathrm{~mm}$ in fig. 6).

The areas close to the plasma are areas of intense erosion. The tungsten marker stripes were almost totally eroded with only very small amounts of tungsten remaining (remaining W was below $9.7 \times 10^{17}$ atoms $/ \mathrm{cm}^{2}$ for $3 \mathrm{X} 11 \mathrm{~L}$ and $4 \times 10^{18}$ atoms $/ \mathrm{cm}^{2}$ for $\left.3 \mathrm{X} 11 \mathrm{R}\right)$. On the $3 \mathrm{X} 11 \mathrm{~L}$ tile this area is $4 \mathrm{~cm}$ wide $(-70 \mathrm{~mm}$ to $-30 \mathrm{~mm}$ in fig. 6$)$; on $3 \mathrm{X} 11 \mathrm{R}$ tile this area is $3 \mathrm{~cm}(0$ $\mathrm{mm}$ to $30 \mathrm{~mm}$ in fig. 6) wide and continues till the end of the tile. The observed large erosion is in qualitative agreement with profiler measurements of the tiles [15], which indicated also a large carbon erosion in these areas.

On the $3 \mathrm{X} 11 \mathrm{~L}$ tile, there is an additional area of deposition in the central part of the tile ($20 \mathrm{~mm}$ to $0 \mathrm{~mm}$ in figure 7), where the IWGL tiles have a small recessed area. The maximum amount of $\mathrm{C}$ detected on the surface of the $\mathrm{W}$ marker stripe is $2 \times 10^{19}$ atoms $/ \mathrm{cm}^{2}$ in this area. Similar central deposition areas have previously been observed in $[14,16]$ and were qualitatively explained by the recess in the central part of the tiles [16].

Between the areas of intense erosion close to the plasma and the areas with little modifications far from the plasma, regions with intense deposition are observed, i.e., $-110 \mathrm{~mm}$ 
to $-80 \mathrm{~mm}$ on tile $3 \mathrm{X} 11 \mathrm{~L}$ and $40 \mathrm{~mm}$ to $100 \mathrm{~mm}$ on tile $3 \mathrm{X} 11 \mathrm{R}$ as shown in figure 7 . Carbon deposits up to $2.3 \times 10^{20}$ atoms $/ \mathrm{cm}^{2}$ on tile $3 \mathrm{X} 11 \mathrm{~L}$ tile and $9.6 \times 10^{19}$ atoms $/ \mathrm{cm}^{2}$ on tile $3 \mathrm{X} 11 \mathrm{R}$ were detected using RBS. Using optical microscopy of tile cross-sections films of up to 48 $\mu \mathrm{m}$ have been observed, see Fig. 7. RBS and optical microscopy show very similar thickness profiles of the deposits. Combining RBS data in atoms $/ \mathrm{cm}^{2}$ and the optical microscopy data in $\mu \mathrm{m}$ it is possible to estimate the density of the deposited film, which is $0.67-0.83 \mathrm{~g} / \mathrm{cm}^{3}$. The major uncertainties that could influence this result are the roughness of the films and of the tile substrate, as well as cross sections used for the spectrum analysis. These uncertainties could account for an error of $0.3 \mathrm{~g} / \mathrm{cm}^{3}$ in the density quoted, indicating that the density of deposits is substantially lower than that of carbon, $2 \mathrm{~g} / \mathrm{cm}^{2}$. The concentration of beryllium in the deposited films was below the detection limit of RBS. Distributions of erosion/deposition areas on limiter tiles with erosion on the plasma-exposed face and deposition on the tile sides was already observed qualitatively in JET [5]. A very similar pattern, with erosion on plasmaexposed limiter faces and re-deposition of eroded material on the sides of limiters and other obstacles was also observed in the limiter tokamak TEXTOR $[17,18]$. The erosion-deposition balance depends on plasma parameters, impurity concentrations, and the detailed geometry with respect to the magnetic field [18], which could induce the qualitatively different erosion/deposition behavior of divertor tiles and IWGL.

The amount of carbon deposited on the surface of the analyzed limiter tiles can be extrapolated to the whole surface area of the IWGLs, assuming the same amount of deposition on all tiles, i.e., 19 tiles on 16 inner limiter beams. During the experimental campaign 2005-2009 a total of $65 \mathrm{~g}$ of carbon is estimated to be deposited on the deposition dominated areas of the IWGL limiters. In comparison profilometry measurements of IWGL tiles give a carbon deposition of $35 \mathrm{~g}$ for the 2007 - 2009 campaigns (assuming an areal density of $1 \mathrm{~g} / \mathrm{cm}^{2}$ for the deposit) in the deposition dominated areas [15]. If the value 
obtained from profilometry is scaled by total plasma duration for the 2007-2009 campaign to 2005-2009 campaign (a factor of 1.78) then the equivalent deposition can be estimated as $62 \mathrm{~g}$ on the IWGL tiles, which is close to the amount of deposition estimated from RBS and cross section SEM measurements. Despite the observed carbon deposition in the deposition dominated areas it should be noted that the IWGL tiles are a net carbon erosion area, because the erosion in the erosion-dominated areas exceeds the deposition in the deposition-dominated areas [15]. The total limiter time in the 2005 - 2009 campaigns, including commissioning and restart periods, was approximately 73200 s. If material eroded from the limiter during the limiter phase is assumed to be locally re-deposited on the edges of the limiters, then a lower estimate of erosion at the inner wall limiter of $8.9 \times 10^{-4} \mathrm{~g} / \mathrm{s}$ can be obtained.

\section{Conclusions}

Erosion of tungsten marker layers and deposition were investigated on JET outer divertor tiles exposed during the discharge period 2007-2009 and on inner wall guard limiter tiles (IWGLs) exposed during the period 2005-2009. Only a local and small erosion of the tungsten coating is observed on the divertor tiles. There are two notable exceptions where intense erosion occurs: (i) on the central area of the load-bearing tile 5 (LBT tile) where approximately $30 \%$ of the initial coating thickness was eroded, and (ii) on the horizontal surface of the tile 8 . In both of these regions the RBS data indicates inhomogeneous erosion of the $\mathrm{W}$ coating, i.e. there is preferential erosion of the tungsten coating from some parts of the surface revealing areas of carbon.

Only small amounts of deposition were observed on the divertor tiles studied. These were the outer divertor edge of the LBT tile where carbon deposition up to $2.6 \times 10^{19}$ atoms $/ \mathrm{cm}^{2}$ was measured and the lower part of tile 7 where $1.5 \times 10^{18}$ atoms $/ \mathrm{cm}^{2}{ }^{13} \mathrm{C}$ from trace gas injection were detected. This is in line with other observations on these tiles. 
A complicated distribution of net erosion and deposition areas is observed on the IWGL tiles. A central area of thin deposition is observed in the recessed part of tile $3 \mathrm{X} 11 \mathrm{~L}$, followed by a strong net erosion area where even the tungsten marker stripe was fully eroded. In the following net deposition area deposits with thicknesses up to $48 \mu \mathrm{m}$ are observed. The total amount of carbon deposited in the deposition-dominated areas of the IWGLs in the experimental campaign of 2005-2009 has been estimated to be $65 \mathrm{~g}$. These findings are in good agreement with results from tile profilometry presented in [15].

\section{Acknowledgements}

This work was supported by EURATOM and carried out within the framework of the European Fusion Development Agreement. The views and opinions expressed herein do not necessarily reflect those of the European Commission.

This work is supported by Russian Ministry of Education and Science 


\section{References.}

[1] M. Mayer et al, J. Nucl. Mater. 363-365 (2007) 101

[2] J.P. Coad et al, J. Nucl. Mater. 363-365 (2007) 287

[3] J P Coad et al, Phys. Scr. T145 (2011) 014003

[4] J. Likonen et al, J. Nucl. Mater. 363-365 (2007) 190

[5] M Mayer et al, Phys. Scr. T81 (1999) 13

[6] M Mayer et al, Phys. Scr. T128 (2007) 106

[7] M. Mayer et al, Nucl. Fusion 47 (2007) 1607

[8] M. Mayer, SIMNRA User's Guide, Report IPP 9/113, Max-Planck-Institut für Plasmaphysik, Garching, Germany, 1997

[9] A.F. Gurbich, Nucl. Instr. Meth. B136-138 (1998) 60

[10] E. Kashy et al Phys. Rev. 122(3) (1961) 884

[11] A.F. Gurbich, Nucl. Instr. Meth. B129 (1997) 311

[12] J.P. Coad et al J. of Nucl. Mater. 390-391 (2009) 992-995

[13] J. Likonen et al this conference

[14] JP. Coad et al. Phys. Scr. T145 (2011) 014003

[15] A. Widdowson et al this conference

[16] M. Mayer et al Physica Scripta. T81(1999) 13-18

[17] P. Wienhold et al, J. Nucl. Mater. 290-293 (2001) 362

[18] M Mayer et al, J. Nucl. Mater. 290-293 (2001) 381

[19] Widdowson, A. Phys. Scr. T138 (2009) 014005 


\section{Figure captions}

Figure 1 Positions of measured divertor tiles, s-coordinates system used in JET divertor and a qualitative map of the distribution of erosion and deposition areas

Figure 2 Remaining W coating thicknesses for LBT14-RW tile after exposure vs. s-cordinate Figure 3 RBS spectrum of an area of intense inhomogeneous erosion, detector counts vs. proton energy

Figure 4 Remaining W coating thicknesses after exposure for tiles G7B and G8A vs. scorrdinate.

Figure 5 Qualitative map of erosion and deposition areas on the pair of inner wall guard limiter tiles

Figure 6 Initial W marker stripe thicknesses (dashed line) and remaining thicknesses after exposure (hollow triangles) vs. the distance to the tile borders along the surface for the left and right inner limiter tiles. Solid lines indicate borders between left and right tiles

Figure 7 Thicknesses of carbon deposits on the W stripe marker after exposure vs. the distance to the tile borders along the surface for the left and right inner limiter tiles. Solid lines indicate borders between left and right tiles. Cross-section data were obtained by optical microscopy 


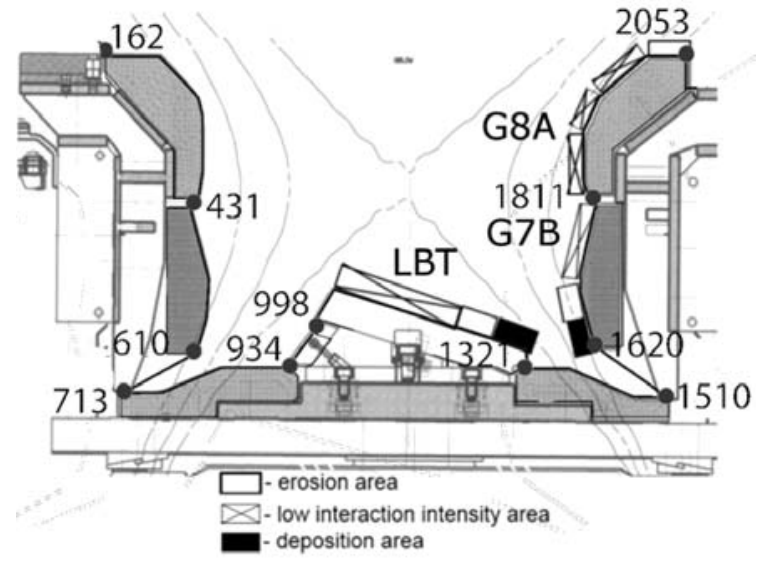

Figure 1 


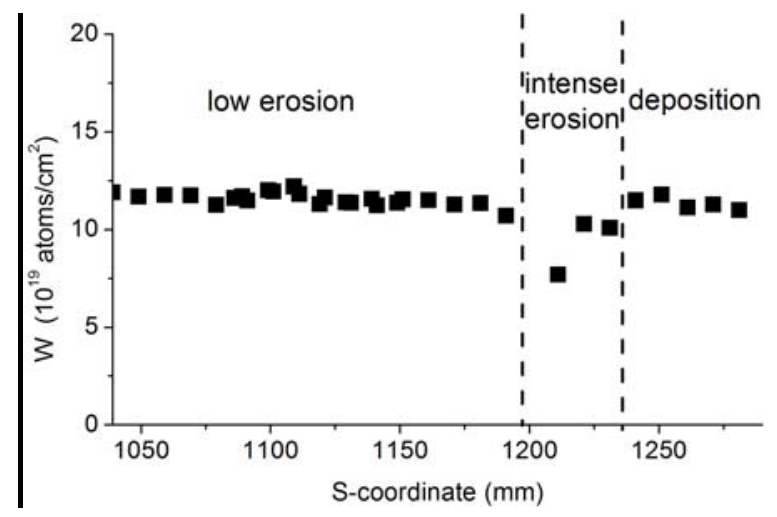

Figure 2 


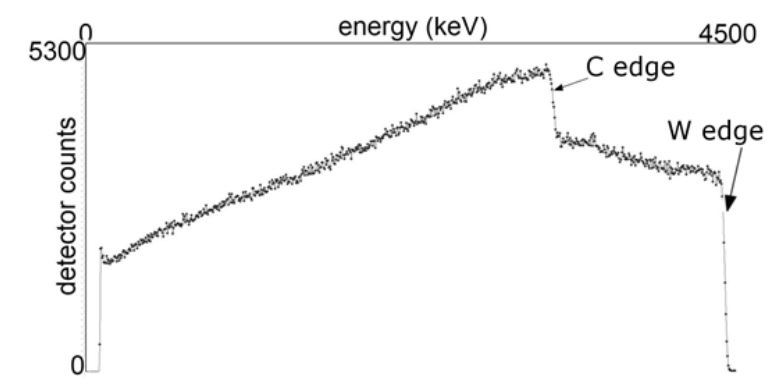

Figure 3 


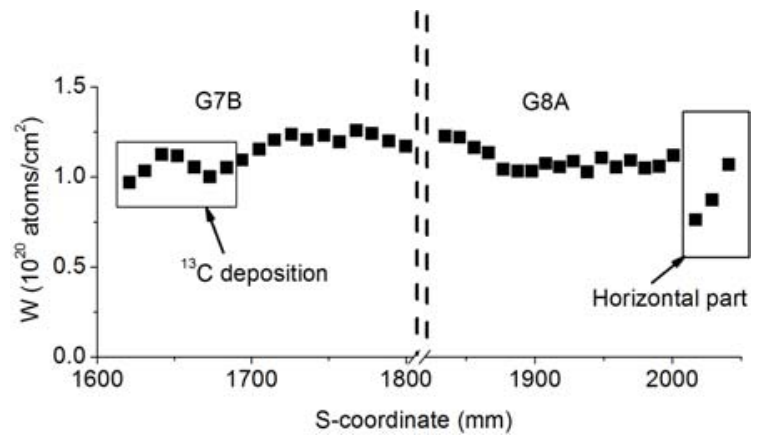

Figure 4 


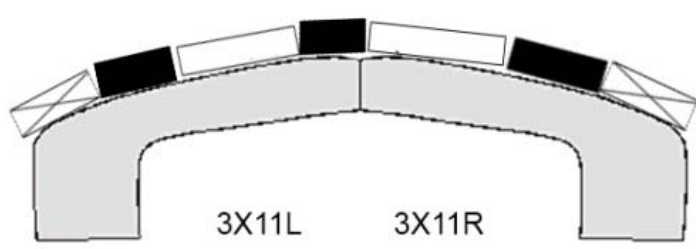

$\square$ - erosion area

$\varangle$ - low interaction intensity area

- deposition area

Figure 5 


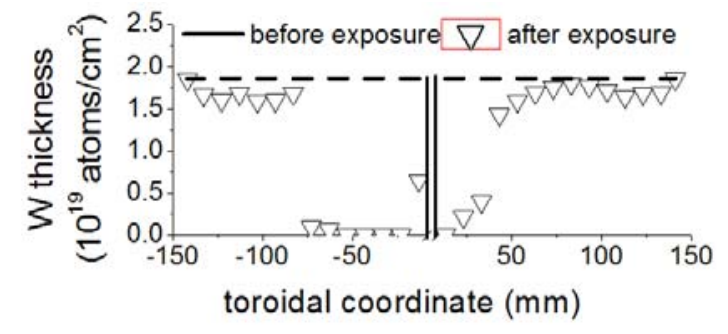

Figure 6 


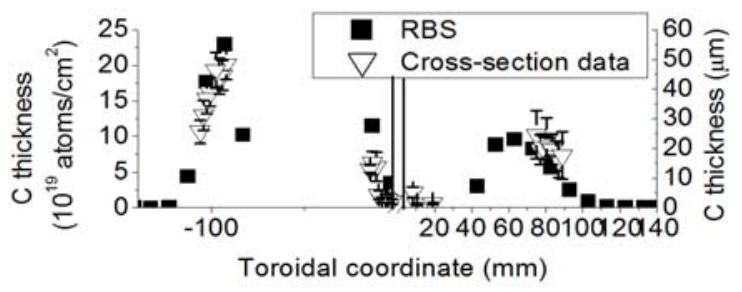

Figure 7 